Accepted for The Astronomical Journal, $\approx$ July, 1996

\title{
K Giants in Baade's Window. II. The Abundance Distribution'
}

\author{
Elaine M. Sadler \\ School of Physics, University of Sydney, NSW 2006, Australia \\ Electronic mail: ems@physics.usyd.edu.au \\ R. Michael Rich ${ }^{2}$ \\ Department of Astronomy, Pupin Laboratories, Columbia University, \\ 538 West 120th Street, New York, New York 10027 \\ Electronic mail: rmr@cuphyd.columbia.edu \\ D. M. Terndrup ${ }^{3}$ \\ Department of Astronomy, The Ohio State University, \\ 174 W. 18th Ave., Columbus, Ohio 43210 \\ Electronic mail: terndrup@baade.mps.ohio-state.edu
}

\begin{abstract}
This is the second in a series of papers in which we analyze spectra of over $400 \mathrm{~K}$ and M giants in Baade's Window, including most of the stars with proper motions measured by Spaenhauer et al. [AJ, 103, 297 (1992)]. In our first paper, we measured line-strength indices of $\mathrm{Fe}, \mathrm{Mg}, \mathrm{CN}$ and $\mathrm{H} \beta$ and calibrated them on the system of Faber et al. [ApJS, 57, 711 (1985)]. Here, we use the $\langle\mathrm{Fe}\rangle$ index to derive an abundance distribution in $[\mathrm{Fe} / \mathrm{H}]$ for 322 stars with effective temperatures between $3900 \mathrm{~K}$ and $5160 \mathrm{~K}$.

Our derived values of $[\mathrm{Fe} / \mathrm{H}]$ agree well with those measured from highresolution echelle spectra (e.g., McWilliam \& Rich [ApJS, 91, 749 (1994)]) for the small number of stars in common. We find a mean abundance $\langle[\mathrm{Fe} / \mathrm{H}]\rangle=-0.11 \pm 0.04$ for our sample of Baade's Window K giants. More than half the sample lie in the range $-0.4<[\mathrm{Fe} / \mathrm{H}]<+0.3$.
\end{abstract}

\footnotetext{
${ }^{1}$ Based on data obtained at the Anglo-Australian Observatory and at the Cerro Tololo Inter-American Observatory, NOAO, which is operated by the Association of Universities for Research in Astronomy, Inc. (AURA), under cooperative agreement with the National Science Foundation.

${ }^{2}$ Alfred P. Sloan Fellow.

${ }^{3}$ Presidential Young Investigator.
} 
We estimate line-of-sight distances for individual stars in our sample and confirm that, in Baade's Window, most $\mathrm{K}$ giants with $V<15.5$ are foreground disk stars, but the great majority (more than 80\%) with $V>16$ belong to the bulge.

We also compare the metallicities derived from the $\mathrm{CN}$ and $\mathrm{Mg}_{2}$ indices to those from iron. Most of the metal-rich stars in our sample appear to be $\mathrm{CN}$-weak, in contrast to the situation in metal-rich globular clusters and elliptical galaxies. The metal-poor half of our sample $([\mathrm{Fe} / \mathrm{H}]<0)$ shows evidence for a mild $\mathrm{Mg}$ overenhancement $([\mathrm{Mg} / \mathrm{Fe}] \sim+0.2)$; but this is not seen in the more metal-rich stars $([\mathrm{Fe} / \mathrm{H}] \geq 0)$. The $\mathrm{K}$ giants in Baade's Window therefore share some, but not all, of the characteristics of stars in elliptical galaxies as inferred from their integrated light.

\section{Subject headings:}

\section{Introduction}

This is the second paper of a series on the radial velocities and metallicities of a large sample of $\mathrm{K}$ giants in the Baade's Window (BW) field of the Galactic nuclear bulge at $(\ell, b)=\left(1^{\circ},-3.9^{\circ}\right)$. In the first paper of this series (Terndrup et al. 1995, hereafter Paper I), we presented line-strength, radial velocity and photometric measurements for over $400 \mathrm{~K}$ and $\mathrm{M}$-giant stars in BW for which proper motions have already been measured (Spaenhauer et al. 1992), and placed the indices on the Lick Observatory system (Faber et al. 1985, hereafter FFBG). The main goals of this paper are to derive the abundance distribution for the $\mathrm{K}$-giant stars in our sample, and to examine the distribution of the stars in distance along the line of sight. Our main abundance indicator is the iron-line index $\langle\mathrm{Fe}\rangle$. We use $V-I$ colors (corrected for extinction) to derive the effective temperature $T_{\mathrm{e}}$ for each star, then apply the empirical calibration derived by FFBG to determine $[\mathrm{Fe} / \mathrm{H}]$ from the measured $\langle\mathrm{Fe}\rangle$. Subsequent papers will discuss the three-dimensional kinematics of the bulge and foreground disk in BW, and present the distribution of metallicities and radial velocities along other lines of sight to the bulge.

The paper is organized as follows: we first (Sec. 2) derive effective temperatures, then determine $[\mathrm{Fe} / \mathrm{H}]$ for individual stars, and finally compare our adopted metallicity scale to those from several previous studies in BW. We then estimate the distances of individual 
stars (Sec. 3) and identify the main population subgroups in the sample. In Sec. 4, we address the question of non-solar abundance ratios in BW stars via the $\mathrm{Mg}$ and $\mathrm{CN}$ indices. We summarize and discuss the implications of our results in Sec. 5.

\section{The Adopted Metallicity Scale}

\subsection{Philosophy}

In Paper I, we tabulated measurements of several of the line-strength indices defined by FFBG and confirmed that our measurements are on the same index system as defined by the standards in that paper. We now need to convert these indices into estimates of $[\mathrm{Fe} / \mathrm{H}]$. We adopt a simple transformation based on the FFBG paper which relies on the $\langle\mathrm{Fe}\rangle$ index, where $\langle\mathrm{Fe}\rangle=0.5 \times(\mathrm{Fe} 5270+\mathrm{Fe} 5335)$, and use a temperature scale given by $V-K$. We later demonstrate that this metallicity scale is in agreement with other estimates in BW, particularly from high-resolution spectra. Alternative approaches for deriving $[\mathrm{Fe} / \mathrm{H}]$ from low-resolution spectra are discussed, for example, by Jones et al. (1995).

Our choice of the FFBG metallicity calibration (which is separate from the calibration of our line-strength indices on the FFBG system) presents several problems. The metallicity calibration, for example, is given by the adopted values of $[\mathrm{Fe} / \mathrm{H}]$ for a large number of field stars, which are based on a sample of standard stars analyzed at various resolutions over many years. This calibration fundamentally rests in turn on analyses of high-resolution spectra of a few of their standard stars. The high-resolution analyses available to FFBG typically did not extend beyond $[\mathrm{Fe} / \mathrm{H}]>0$; the few stars at very high abundances typically had estimates based on low-resolution spectra or narrow-band photometry which centered on one or two spectral features, particularly CN. Many of these analyses were done 30-40 years ago, and there has been considerable progress since then in the study of field-star abundances. Similarly at low-metallicities, the line-strength indices become very weak and the derived metallicities have relatively high errors. It is therefore possible that future work may revise the metallicity scale of the Lick indices and thus our metallicity calibration for our sample.

Another limitation of the FFBG system is that, even with subsequent extensions and modifications, there are indications that their standards to not cover the full range of temperature, metallicity, gravity, and non-solar abundance ratios which may be present throughout the Galaxy. The original FFBG calibration was extended significantly by 
Gorgas et al. (1993, hereafter G93), who increased the number of standards in the system and placed special emphasis on exploring metallicity and gravity effects. They computed polynomial "fitting functions" which described the behavior of the FFBG indices as a function of temperaturef (parameterized by $V-K$ ), gravity $(\log g$ ), and metal abundance $(Z \equiv[\mathrm{Fe} / \mathrm{H}])$. They showed that the Fe 5270 and Fe 5335 indices were sensitive to abundance but insensitive to gravity, while the $\mathrm{Mg}$ indices were highly sensitive to both metallicity and gravity. G93 described a procedure for deriving $Z$ and $\log g$ for individual stars by averaging estimates from the $\mathrm{CN}, \mathrm{Mg}_{2} \mathrm{Fe} 5270$, and Fe 5335 indices. For data with good signal-to-noise, this is accurate to about 0.25 dex in $Z$ and $0.23 \operatorname{dex}$ in $\log g$. Despite increasing evidence for non-solar abundance ratios in different galactic populations, G93 concluded that only one metallicity parameter was needed to characterize abundance variations among the different populations of stars in the library. They reached this conclusion by demonstrating that the scatter about the (three parameter) fitting functions was only slightly larger than the errors of measurement, i.e., that there was no remaining variation which required the introduction of other parameters, particularly those relating to non-solar abundances ratios such as $[\mathrm{Mg} / \mathrm{Fe}]$.

We decided to adopt the simpler FFBG approach, which relies primarily on the $\langle\mathrm{Fe}\rangle$ index, for several reasons. The first reason was that the FFBG calibrations use simple linear or quadratic equations, which are easier to compute than the more complicated fitting functions in G93. The $\langle\mathrm{Fe}\rangle$ index itself, furthermore, is insensitive to gravity (G93), which means that it is not necessary to know the distances to the individual stars for an accurate estimate of $[\mathrm{Fe} / \mathrm{H}]$. In addition, we presented evidence in Paper I that many stars in Baade's Window have stronger $\mathrm{Mg}_{2}$ absorption relative to $\langle\mathrm{Fe}\rangle$ than stars of the same color in the G93 libraries. We interpreted this to mean that if the Baade's Window giants were relatively old $\left(t>8 \times 10^{9} \mathrm{yr}\right)$, they have an enhanced relative abundance in $\mathrm{Mg}$ (i.e., $[\mathrm{Mg} / \mathrm{Fe}]>0)$. In this case, an abundance measurement using a combination of $\mathrm{Mg}$ and $\mathrm{Fe}$ lines would yield a higher metallicity than one which used Fe alone (cf. McWilliam \& Rich 1994). Equivalently, if the metallicity scale from the iron and magnesium lines were equal (as in G93), then for moderate distances from the Sun ( $R \sim 8 \mathrm{kpc}$ ) they would be more massive than is allowed from studies of the main-sequence turnoff. Since one of the aims of this paper is to explore the possibility of non-solar abundance ratios (below, Sec. 4), we wished to avoid the implied equality between the $\mathrm{Mg}$ and Fe scales in G93. Finally, the errors in our measured values of $\langle\mathrm{Fe}\rangle$ for individual stars are often too large for us to set

\footnotetext{
${ }^{4}$ Worthey et al. (1994) presented a modification to the G93 approach, in which the effective temperature itself (specifically $\Theta_{e}=5040 / T_{\mathrm{e}}$ ) was used as the temperature parameter in fitting functions, rather than $V-K$.
} 
effective constraints on the two free parameters $([\mathrm{Fe} / \mathrm{H}]$ and $\log g)$ in the G93 formalism.

We observed more than twenty of the abundance standard stars listed by FFBG, but (as described in Paper I) these observations were used only to transform our line-strength measurements of the BW stars to the Lick system. We were able to place our index values accurately on the FFBG system. For the eight standards (excluding HR 5270) observed at the AAT, the mean difference between our derived abundance $[\mathrm{Fe} / \mathrm{H}]$ and the FFBG value was $(\mathrm{AAT}-\mathrm{FFBG})=-0.02 \mathrm{dex}$, with a dispersion of 0.27 dex. For the much larger sample of standards observed at CTIO, the difference (CTIO - FFBG) was +0.06 dex, with a dispersion of 0.25 dex. (The Fe 5270 and Fe 5335 indices were transformed to the FFBG system separately, then averaged.)

\subsection{The Temperature Scale}

The first step deriving metallicities for the BW sample is to transform each $V-I$ color to an effective temperature $T_{\mathrm{e}}$. Several authors have investigated the metallicity and gravity dependence of the transformation between broad-band colors and $T_{\mathrm{e}}$, and have generally found similar relations between effective temperature and $V-I$ agree well (e.g., Worthey et al. 1994, their Figure 2) at least for $T_{e}>3800 \mathrm{~K}$. For this study, we used an empirical temperature calibration of the Cousins VRI system (Bessell 1979), and calculated $V-I$ colors for a grid of models of known temperature (Bell \& Gustafsson 1989). These two scales are similar, though the models are 0.08 mag bluer than Bessell's values for solar-metallicity giants at the cool end of the $\mathrm{K}$ giant temperature distribution $\left(T_{\mathrm{e}} \approx 4000\right.$ $\mathrm{K})$. Here we adopt the Bessell relation between the reddening-corrected color $(V-I)_{0}$ and $T_{\mathrm{e}}$, which is displayed in Figure 1.

We restrict our derivation of effective temperatures to the $K$ stars in our sample, because the relation between $V-I$ and $T_{\mathrm{e}}$ is essentially independent of surface gravity for $\mathrm{K}$ stars (Bessell 1979), but not for the cooler M stars. The relation between temperature and broad-band colors, as derived from model fluxes, is also largely independent of metallicity for $T_{\mathrm{e}}>3800 \mathrm{~K}$ (e.g., Bessell et al. 1989; Worthey et al. 1994 and references therein). For the cooler $\mathrm{M}$ giants the temperature scale is increasingly poorly defined, because of TiO blanketing in the $V$ band and a lack of calibration stars with reliably-determined temperatures (e.g., Ridgway et al. 1980). We therefore chose to restrict our analysis to stars with $T_{\mathrm{e}}$ in the range $3900-5160 \mathrm{~K}$.

Aside from random errors in the photometry, there are two sources of error in the $V-I$ colors and the determination of effective temperatures. These were extensively discussed 
in Paper I. The first systematic error arises from uncertainties in the zero points of the photometry on the several CCD frames from which colors were measured. This error is of order $\approx 0.04 \mathrm{mag}$ on each frame. The second error comes from the uncertainty in the adopted reddening. In paper $\mathrm{I}$, we derived an average reddening of $\mathrm{E}_{V-I}=0.64 \pm 0.08$ mag for the entire BW sample, and showed that there is marginal evidence in our data for variations in the reddening across the field. The BW field has large (and patchy) reddening (e.g., Figure 1 of Blanco 1984); so the average extinction value may not be appropriate for the individual stars in our sample. If we have overestimated the reddening to an individual star then we will derive a temperature which is too hot and an abundance which is too high. Similarly, underestimating the reddening will yield a temperature which is too cool and an abundance estimate which is too low. We discuss the effects of these uncertainties at greater length below.

\subsection{Computation of $[\mathrm{Fe} / \mathrm{H}]$}

The next step in computing metallicities was to recast the FFBG empirical calibration of the $\langle\mathrm{Fe}\rangle$ index, which represents temperature by $V-K$ color, into expressions in $T_{\mathrm{e}}$. We converted the $V-K$ colors listed by FFBG to $T_{\mathrm{e}}$ using stars whose effective temperature is tabulated by Bell \& Gustafsson (1989). This allowed us to rewrite the FFBG calibration in terms of $T_{\mathrm{e}}$ rather than $V-K$, and hence to apply it to the BW stars since we have no $V-K$ photometry for most of the sample.

For reasons discussed above, we then restricted our computation of $[\mathrm{Fe} / \mathrm{H}]$ to those stars with $T_{\mathrm{e}}$ between $3900 \mathrm{~K}$ and $5160 \mathrm{~K}$, corresponding to spectral types between $\mathrm{K} 5$ and G8. (The FFBG calibration is defined for a slightly wider temperature range, roughly 3800-5200 K.) Of the 432 stars listed in Paper I, 369 have $V-I$ colors which place them in our restricted temperature range. Of the remaining 63 stars, 36 are cooler ( $\mathrm{M}$ stars), 16 are hotter (G stars), and we have no photometry for 11. We were able to measure $\langle\mathrm{Fe}\rangle$ for 322 of these 369 stars. For the remainder, we either have no spectrum or the $\langle\mathrm{Fe}\rangle$ index was unmeasurable because of a poor signal-to-noise ratio in that region of the spectrum or (for the CTIO spectra) a cosmic-ray hit.

\footnotetext{
${ }^{5}$ Alternatively, we could have used the empirically derived relation between $V-I$ and $V-K$ for BW stars (Tiede et al. 1995; Paper I) and used the FFBG calibration directly. Experiments showed that this approach yielded nearly identical results to the one we adopted here.
} 
For these 322 stars, we used $T_{\mathrm{e}}$ and the iron line strength $\langle\mathrm{Fe}\rangle$ to derive a metal abundance $[\mathrm{Fe} / \mathrm{H}]$ using our recast FFBG formulation. FFBG's Table 4 gives the relation between $V-K,[\mathrm{Fe} / \mathrm{H}]$ and a parameter $\langle\Delta \mathrm{Fe}\rangle$, defined as the difference between the observed $\langle\mathrm{Fe}\rangle$ and the value expected for a star of solar abundance at the same temperature. In Figure 2 we plot this in a slightly different form, showing $\langle\mathrm{Fe}\rangle$ versus $T_{\mathrm{e}}$ for a range of values of $[\mathrm{Fe} / \mathrm{H}]$, along with the observed values.

Table 1 lists all the BW stars in our sample for which we were able to measure $[\mathrm{Fe} / \mathrm{H}]$. The errors in $[\mathrm{Fe} / \mathrm{H}]$ were initially estimated from the errors in the individual values of $\langle\mathrm{Fe}\rangle$ listed in Paper I. However, a cross-comparison of $[\mathrm{Fe} / \mathrm{H}]$ for stars in common with previous bulge studies (immediately below) suggested that our errors were overestimated by almost a factor of two. We have therefore adjusted our original estimates to give the final errors quoted in Table 1.

The FFBG relations are only valid for stars with $[\mathrm{Fe} / \mathrm{H}]$ from -0.8 to $\sim+0.5$. The FFBG system loses sensitivity at low metallicities because the Fe features become weak; while the high abundances may be in error because they are derived by extrapolating past the calibrated metallicities (this is a separate issue from the validity of the adopted metallicities in the FFBG system at high $[\mathrm{Fe} / \mathrm{H}]$ ). About $7 \%$ of the stars in our sample have $[\mathrm{Fe} / \mathrm{H}]$ below this range, and $14 \%$ have abundances above. For these stars, the derived $[\mathrm{Fe} / \mathrm{H}]$ values are likely to be indicative only.

\subsection{Error Estimates and Comparison with Other Studies}

We have a number of stars in common with other studies of the metallicity distribution in BW. Figure 3 shows the correlation between our $[\mathrm{Fe} / \mathrm{H}]$ estimates and those from (top panel) Washington photometry by Geisler \& Friel (1992; hereafter GF92) and (lower panel) low-resolution spectra by Rich (1988, hereafter R88). Our [Fe/H] measurements are systematically lower than those from R88 and GF92 by 0.19 and 0.15 dex respectively (see Table 2 for a summary). The difference appears to be a simple zero-point shift, independent of temperature and abundance (Figure 3). Table 3 gives a cross-identification for the GF92 stars in common with our sample.

Recently, McWilliam \& Rich (1994, hereafter MR94) have presented a detailed analysis of high-resolution spectra of $11 \mathrm{~K}$-giants in BW, six of them in common with our sample. Consistent with our findings above, they showed that their metallicity scale was lower on average than that of R88 and GF92 by $\approx 0.3$ dex. They discuss the reasons for this 
offset in some detail, and conclude that the most likely explanation for the previous higher metallicity scale is that the earlier work was measuring something more like $[(\mathrm{Fe}+\mathrm{Mg}) / \mathrm{H}]$, which would be higher than $[\mathrm{Fe} / \mathrm{H}]$ if $[\mathrm{Mg} / \mathrm{Fe}]>0$. Denoting our metallicities from Table 1 by SRT, we find that $\left\langle[\mathrm{Fe} / \mathrm{H}]_{\mathrm{SRT}}-[\mathrm{Fe} / \mathrm{H}]_{\mathrm{MR} 94}\right\rangle=+0.02 \pm 0.29$ (s.d.), showing that the simple FFBG metallicity estimates we adopt here agree well with those derived by MR94 for the stars in common.

Another study at high resolution is by Bessell et al. (1995), who obtained echelle spectra of seven K giants in BW, six of which are in common with our study and one in common with MR94. The mean difference in $[\mathrm{Fe} / \mathrm{H}]$ (in the sense SRT - Bessell et al.) is $+0.15 \pm 0.18$. Combining the results from the Bessell et al. (1995)] study and MR84, we find a mean difference of $+0.12 \pm 0.22$ (s.d.) for the 11 stars in common with both echelle studies (the scale here is the higher).

We have no stars in common with the study of Castro et al. (1995), who obtained high-resolution spectra of two stars in BW, and who also concluded that the metallicity scale from Rich (1988) was too high by a few tenths dex.

Figure 4 shows the correlation between our abundances and those from both highresolution studies. There is some indication that the our metallicities are too high for $[\mathrm{Fe} / \mathrm{H}]$ $>+0.5$, which is not surprising since these were generated with a long extrapolation from the metallicities present in the FFBG libraries. Since the computed mean differences are statistically equal to zero, however, we conclude that the simple FFBG calibration of the $\langle\mathrm{Fe}\rangle$ index gives a metallicity scale consistent with the currently available high-resolution studies in BW, and make no further adjustment to the abundances listed in Table 1.

We can use the stars in common with R88 and GF92 to estimate the true (external) errors in $[\mathrm{Fe} / \mathrm{H}]$ for our sample. We originally estimated a mean error of 0.44 dex for our $[\mathrm{Fe} / \mathrm{H}]$ measurements from propagation of errors in the $\langle\mathrm{Fe}\rangle$ index, but we reduced this by a factor of 0.55 to achieve consistent values of the errors in the three studies. R88 and GF92 have seven stars in common, for which

$$
\sigma_{(\mathrm{R} 88-\mathrm{GF} 92)}=0.245 \mathrm{dex}
$$

where $\sigma$ is the dispersion in $\Delta[\mathrm{Fe} / \mathrm{H}]=[\mathrm{Fe} / \mathrm{H}]_{\mathrm{R} 88}-[\mathrm{Fe} / \mathrm{H}]_{\mathrm{GF} 92}$. From Table 2:

$$
\begin{aligned}
\sigma_{(\mathrm{SRT}-\mathrm{R} 88)} & =0.286 \mathrm{dex}(48 \text { stars }) \\
\sigma_{(\mathrm{SRT}-\mathrm{GF} 92)} & =0.301 \mathrm{dex}(42 \text { stars })
\end{aligned}
$$

after excluding the three discrepant stars noted in Table 3. Assuming $\sigma_{(\mathrm{SRT}-\mathrm{R} 88)}^{2}=\sigma_{\mathrm{SRT}}^{2}+\sigma_{\mathrm{R} 88}^{2}$, where $\sigma_{\mathrm{SRT}}$ and $\sigma_{\mathrm{R} 88}$ are respectively the errors in the 
SRT and R88 measurements of $[\mathrm{Fe} / \mathrm{H}]$, we find:

$$
\begin{aligned}
\sigma_{\mathrm{R} 88} & =0.16 \mathrm{dex} \\
\sigma_{\mathrm{GF} 92} & =0.19 \mathrm{dex} \\
\sigma_{\mathrm{SRT}} & =0.24 \mathrm{dex}
\end{aligned}
$$

These estimates agree well with the mean errors quoted by R88 and GF92 for their own data, which are 0.18 dex and 0.25 dex respectively. The mean of the adjusted errors in Table 1 (reduced by a factor 0.55 from the original estimates) is 0.24 dex. It is probably not surprising that the mean error in our $[\mathrm{Fe} / \mathrm{H}]$ measurements is slightly higher than that of R88. Most of our stars are fainter than those observed by R88, and the errors reflect the increased effects of crowding as we go fainter.

\subsection{Reddening and the abundance calibration}

We now examine the effects of reddening on our abundance measurements, and discuss whether different assumptions about the reddening to BW might account for some of the difference between our abundance measurements and those of R88 and GF92.

We consider three questions:

(i) how do different assumptions about the mean reddening in BW affect the derived abundance distribution?

(ii) is our assumption of a single reddening for the whole BW sample reasonable?

(iii) what are the effects of patchy reddening for individual stars?

By examining the dependence of $[\mathrm{Fe} / \mathrm{H}]$ on temperature in the FFBG calibration, we find empirically that the effect of small changes in $\mathrm{E}_{V-I}$ on the derived value of $[\mathrm{Fe} / \mathrm{H}]$ varies with temperature, and is generally larger for hotter stars. Figure 5 shows the relation between $\delta[\mathrm{Fe} / \mathrm{H}] / \mathrm{E}_{V-I}$ and $T_{\mathrm{e}}$ for $\mathrm{K}$ giants in our sample. The average change in $[\mathrm{Fe} / \mathrm{H}]$ is 0.15 dex per $0.1 \mathrm{mag}$ in color, with a maximum value of 0.2 . In Paper I, we estimated the error in the average reddening for the entire sample to be 0.08 mag. The maximum effect of a change of $\pm 0.08 \mathrm{mag}$ in the adopted reddening would be a shift of \pm 0.16 dex in $[\mathrm{Fe} / \mathrm{H}]$ (for stars with $T_{\mathrm{e}} \sim 4600 \mathrm{~K}$ ), which is somewhat smaller than the uncertainty in an individual measurement. (Increasing the reddening would increase $T_{\mathrm{e}}$, and since $\langle\mathrm{Fe}\rangle$ remains constant, the derived value of $[\mathrm{Fe} / \mathrm{H}]$ would also increase.)

Could part of the zero-point shift between our data and that of R88 and GF92 be due to differences in the assumed reddening to Baade's Window? Both GF92 and MR94 
adopted essentially the same reddening as we did $\left(\mathrm{E}_{V-I}=0.64 \mathrm{mag}\right.$; equivalent to $\mathrm{E}_{(B-V), 0}$ $=0.52 \mathrm{mag}) . \mathrm{R} 88$, however, adopted a slightly higher reddening $\left(\mathrm{E}_{(B-V), 0}=0.56 \mathrm{mag}\right)$ and so would be expected to derive a higher abundance for any given star. It is difficult to estimate directly the effect of the higher reddening in the R88 study, since Rich used $J-K$ rather than $V-I$ colors to measure temperature, and conversion between the two is not straightforward. If we had adopted the higher value of $\mathrm{E}_{(B-V), 0}=0.56$ mag, our abundances would be higher on average by 0.06 dex. This is considerably smaller than the measured difference in the metallicity scales (0.19 dex), from which we conclude that the differences between our values and those of R88 and G92 does not simply arise from differences in the adopted reddening to BW. MR94 discuss the zero-point of the R88 scale in considerable detail, and conclude that the R88 scale measures not only the Fe abundance but that of $\mathrm{Mg}$ as well, which may be elevated in bulge stars. This may also be true of the G92 scale, which measures essentially the total absorption from all lines in the blue-yellow portion of the spectrum, including the strong $\mathrm{MgH}$ and $\mathrm{Mg} b$ features.

In Paper I we also showed that there was a possible variation in the extinction (on large angular scales) up to \pm 0.05 mag across our BW field. As discussed above, this should not produce a significant error in the metallicity scale in different parts of the sample. We tested this by computing the mean $[\mathrm{Fe} / \mathrm{H}]$ in the parts of BW which Blanco et al. (1984) identified as having different extinctions. In their region $\mathrm{A}$, we find $\langle[\mathrm{Fe} / \mathrm{H}]\rangle=-0.11 \pm 0.04$, while in the combined regions $\mathrm{BC}$ we measure $\langle[\mathrm{Fe} / \mathrm{H}]\rangle=-0.12 \pm 0.05$. The equality in the two mean values suggests that there are no significant systematic errors in our metallicity scale which depend on the positions within BW.

Patchy reddening in the BW field will also affect the abundances we measure for individual stars. The general impression from deep photographs of BW (e.g., Figure 1 of Blanco 1984) is that some individual stars may have an extinction which is considerably different from the average. Since we cannot estimate the extinction towards individual stars in our sample, we are unable to determine the variation in the extinction towards BW on small angular scales.

\section{The line--of-sight to Baade's Window}

The derivation of distances to individual stars in BW is essential to the interpretation of their proper motions. The only selection criteria for inclusion in the BW proper motion sample were that the stars be relatively uncrowded, and brighter and redder than certain magnitude limits (Spaenhauer et al. 1992). 
As discussed in Paper I, we expect that our sample will include some foreground stars in the old disk or thick disk, especially at the bright end. This was indeed seen in the data: the BW stars with $V<15.5$ have a lower velocity dispersion, a non-zero mean proper motion in Galactic latitude, and a disk-like anisotropy in velocity dispersion, consistent with that expected for foreground disk stars at intermediate distances (Spaenhauer et al. 1992; Paper I). In this section, we derive distances for the stars in our sample, look for additional signatures of the presence of foreground stars, and provide several arguments for the plausibility of our approach.

\subsection{What do we expect to see?}

What is the likely brightness of bulge and disk giants in Baade's Window? Figure 6 shows the predicted observed $\mathrm{V}$ magnitude, $\mathrm{V}_{\text {pred }}$, for $\mathrm{K} 0$ and $\mathrm{K} 5$ giants (and a $\mathrm{K} 0$ dwarf) of roughly solar abundance as a function of distance from the Sun, using absolute magnitudes $\mathrm{M}_{V}$ from Mihalas \& Binney (1981) and assuming a constant extinction of $\mathrm{A}_{V}$ $=1.60 \mathrm{mag}$ along the line of sight. While this is clearly a simplification, it gives us a rough idea of what we expect to see in our sample.

We first conclude that our sample is unlikely to contain many disk dwarfs. Unless these stars are within $1 \mathrm{kpc}$ of the Sun, they will fall below the magnitude cutoff of the proper motion sample $(V \sim 17.5)$. Because of Galactic rotation, however, dwarfs within 1 $\mathrm{kpc}$ of the Sun would be expected to show a high proper motion 1 in $l$ relative to the rest

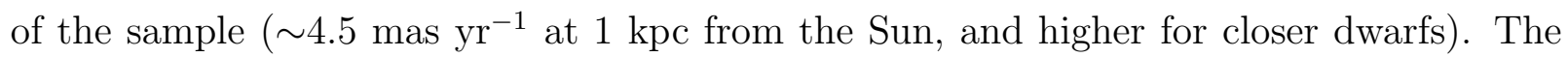

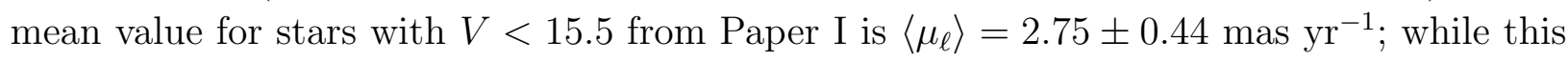
is non-zero, as expected for foreground stars, it is considerably smaller than expected for stars with distances less than $1 \mathrm{kpc}$. We therefore conclude that our sample is unlikely to contain many foreground dwarfs and that most stars located in front of the bulge will be giants in the disk.

If the Galactic Center distance is $R_{0}=7.5 \mathrm{kpc}$ and the bulge has a radius of $1-2 \mathrm{kpc}$, then bulge giants will have typical apparent magnitudes of $\mathrm{V} \sim 15-17$. Disk giants closer than $5 \mathrm{kpc}$ will have $V$ in the range $12-16$. Disk giants on the far side of the bulge would

\footnotetext{
${ }^{6}$ The zero point of the proper motion sample is unknown, so Spaenhauer et al. (1992) set $\left\langle\mu_{\ell}\right\rangle=0$, $\left\langle\mu_{b}\right\rangle=0$. Thus if most of the BW sample is near the bulge, disk stars will show a proper motion about equal in magnitude to the true reflex solar motion of the galactic center. At $R=7.5 \mathrm{kpc}$, the Galactic orbital

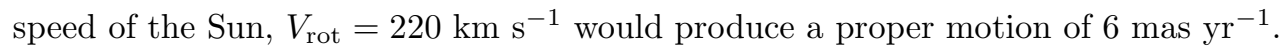


have $V>16-17$. We do not, however, expect to see many distant stars in the old disk since the line-of-sight to BW is $\sim 700$ pc below the disk at $10 \mathrm{kpc}$ from the Sun; stars at these distances may be in the halo or thick disk as we discuss below.

Figure 6 suggests that most stars fainter than $V \sim 15.5$ are likely to be in the bulge and most stars brighter than this are likely to be in the foreground disk, at least for giants of roughly solar abundance. The large abundance spread in BW provides an extra complication, since stars of higher abundance will be fainter than stars of lower abundance at constant color. For $V \sim 15-16.5$, therefore, we expect to see a mix of bulge and disk stars. We now consider how to identify these individually.

\subsection{Distance estimates}

We can derive a photometric parallax for each star by comparing its $I$ magnitude to the absolute magnitude $M_{\mathrm{I}}$ of a globular cluster giant of the same $(V-I)_{0}$ and $[\mathrm{Fe} / \mathrm{H}]$. The lefthand panel of Figure 7 shows the mean locus in $(V-I)_{0}, \mathrm{M}_{\mathrm{I}}$ of the giant branches of four globular clusters with good-quality color-magnitude diagrams (CMDs). These clusters span a range in $[\mathrm{Fe} / \mathrm{H}]$ which overlaps the lower half of metal abundances of the bulge. They are (from blue to red) NGC $6397([\mathrm{Fe} / \mathrm{H}]=-1.9)$, NGC $1851([\mathrm{Fe} / \mathrm{H}]=-1.3)$ and 47 Tuc $([\mathrm{Fe} / \mathrm{H}]=-0.7)$ from Da Costa \& Armandroff (1990), plus the metal-rich globular cluster NGC 6553 (Ortolani et al. 1990) for which we adopt $[\mathrm{Fe} / \mathrm{H}]=-0.2, \mathrm{E}_{V-I}=0.77$ mag, $\mathrm{A}_{\mathrm{I}}=1.42 \mathrm{mag}$. and a distance of $4.9 \mathrm{kpc}$ (Barbuy et al. 1992; Bica et al. 1991).

For each star in our sample, we interpolated between the cluster CMDs in Figure 7 to determine the absolute magnitude $M_{I}$ corresponding to the spectroscopically-determined abundance $[\mathrm{Fe} / \mathrm{H}]$. Comparing $\mathrm{M}_{\mathrm{I}}$ with the dereddened $I_{0}$ magnitude then gives a distance modulus for each star. For stars with $[\mathrm{Fe} / \mathrm{H}]>-0.2$ (about half our sample), we had to extrapolate from the NGC $6553 \mathrm{CMD}$, since photometry in $V I$ for more metal-rich clusters is not available or comes from clusters with significant uncertainty in the reddening or distance modulus. In the righthand panel of Figure 7, we plotted the four cluster giant branches (thin solid lines), along with a giant branch (thick solid line) computed as an extrapolation from NGC 6553. The adjacent dotted line to that extrapolated giant branch is the sequence at $[\mathrm{Fe} / \mathrm{H}]=+0.3$ and an age of 10 Gyr from the Revised Yale Isochrones (Green et al. 1987). The nearby points $(\times)$ display the mean giant branch from the photometry of the metal-rich old open cluster NGC 6791 (Montgomery et al. 1994: Kaluzny \& Rucinski 1995) which has been plotted using the values of reddening and distance modulus advocated for this cluster by Trippico et al. 1995, who derive $[\mathrm{Fe} / \mathrm{H}]$ 
$=+0.36 \pm 0.12$ (we prefered not to use the cluster photometry directly in our computation of distances because the available data extends only $\sim 1$ mag above the cluster horizontal branch). The giant branches of NGC 6791, from the RYI, and as extrapolated in our computation agree on average to a few hundredths mag in $M_{I}$, suggesting that up to this metallicity our computation of distances does not contain significant systematic errors. Beyond $[\mathrm{Fe} / \mathrm{H}]=+0.3$, of course, the extrapolation is uncertain and any distance estimate must be regarded with caution.

\subsection{The Bulge and the Disk}

We now show that our sample contains subgroups which may be identified with giant-branch and clump stars in the bulge, giants in the foreground disk, and a few stars from the halo beyond the bulge. Our aim here is to identify likely bulge stars in a way which is independent of their kinematics. In the solar neighborhood, the two main stellar populations (the disk and halo) can be distinguished either by abundance or by kinematics, but it is not possible to separate the bulge and disk populations in Baade's Window (BW) in the same way. The mean abundance of $\mathrm{K}$ giants in BW is similar to that of of the local disk (MR94), so we cannot reliably distinguish between bulge and disk stars on the basis of abundance. Radial velocity measurements by themselves are not very helpful either bulge stars have a high velocity dispersion $\left(\sim 110 \mathrm{~km} \mathrm{~s}^{-1}\right)$, while the velocity dispersion of the old disk along the line-of-sight to BW increases from $40 \mathrm{~km} \mathrm{~s}^{-1}$ near the Sun to $\sim 90$ $\mathrm{km} \mathrm{s}^{-1}$ near the Galactic center (Lewis \& Freeman 1989).

We do not intend in the following discussion to imply that every star can be unambiguously placed into distinct population bins; rather we argue that the combination of metallicities and distances which we have derived allows us to identify the principal subgroups of our sample in a way which is consistent with current information about the structure and kinematics of the Milky Way. The numerical values we derive below for metallicities and velocity dispersions are summarized in Table 4.

\subsubsection{Clump/RHB stars in the bulge}

We first note that the photometric parallax method we describe above may be systematically in error for the clump or red horizontal branch (RHB) giants which are 
numerous in BW (Terndrup 1988; Paczynski et al. 1994, hereafter P94; Tiede et al. 1995). Clump giants appear on CMDs of the bulge at somewhat bluer colors than the center of the giant branch (equivalently, at brighter magnitudes at each color), so distances estimated from their photometric parallax may be systematically low. We used the location of the RHB/clump in 47 Tuc and NGC 6553 to estimate the region of the CMD in BW where most of the clump/RHB stars lie. To allow for the distance spread within the bulge, we took a range of $\mathrm{M}_{\mathrm{I}}(7.5)-0.8$ to +0.1 for the bulge clump/RHB stars, where $\mathrm{M}_{\mathrm{I}}(7.5)$ is the magnitude each star would have at a distance of $7.5 \mathrm{kpc}$. Note that these stars would be either clump stars or first-ascent giants in the vicinity of the clump. It is also possible that this narrow magnitude range contains a few disk giants.

On this basis, we estimate that $196 / 322$, or $61 \%$ of our sample, are clump/RHB giants. Most of these stars have $V$ between 16.5 and 17.5, which is the location of the clump on our CMDs (Figure 1 of Paper 1). This appears at first to be a very high fraction, but P94 also note that red clump stars outnumber red giant-branch stars in their photometric samples of several BW fields. P94 use slightly different criteria to distinguish clump and giant-branch stars, but the results are very similar: applying their selection criteria (1) and (2) to our sample gives $222 / 322$, or $69 \%$, clump stars. We measure a weighted velocity dispersion (using the prescription in Armandroff \& DaCosta 1986) of $111 \pm 13 \mathrm{~km} \mathrm{~s}^{-1}$ (see Table 4), which agrees well with that measured for other stellar samples in Baade's Window (Rich 1990; Sharples et al. 1990), and $\mathrm{SiO}$ masers in the same region of the bulge (Eumiura $e$ e al. 1995). Thus the group we identify as clump/RHB stars appears kinematically similar to other bulge populations. W

\subsubsection{Giants in the Bulge}

Since the Galactic bulge is spatially extended, stars in the bulge will span a range in distance. We take $\sim 1 \mathrm{kpc}$ as the characteristic radius of the Galactic nuclear bulge, based on the distribution of M giants (Blanco \& Terndrup 1989), IRAS point sources (Habing et al. 1985; Harmon \& Gilmore 1988) and the infrared surface brightness of the bulge (Kent 1992; Weiland et al. 1994). Thus we would expect our distance measurements for bulge giants to span a range of at least $R=7.5 \pm 1 \mathrm{kpc}$. There will, however, be an extra spread due to distance errors. The mean error in $[\mathrm{Fe} / \mathrm{H}]$ for an individual star in our sample is $\sim 0.24$ dex, which corresponds to $\sim \pm 0.3 \mathrm{mag}$ in the distance modulus. A realistic distance spread (taking into account both the depth of the bulge and the uncertainty in determining $[\mathrm{Fe} / \mathrm{H}]$ from the measured $\langle\mathrm{Fe}\rangle)$ is therefore $7.5 \pm 2.5 \mathrm{kpc}$, so that stars with $R$ in the range 
5-10 kpc are likely to be bulge giants. Of the 126 stars in Table 1 which are not clump stars, 72 fall into this class. They have a mean distance of $7.0 \mathrm{kpc}$, and a median distance of $7.1 \mathrm{kpc}$. The velocity dispersion for this group is $95 \pm 17 \mathrm{~km} \mathrm{~s}^{-1}$, slightly lower than but equal to within the errors to the value for the clump/RGB subsample.

\subsubsection{Foreground disk giants}

We next identify as stars in the foreground disk as those which have an estimated distance $\mathrm{R}<5 \mathrm{kpc}$, and which are not clump/RHB stars. There are 39 such stars (12\% of our sample), with a mean distance of $3.3 \mathrm{kpc}$ from the sun.

The radial velocity dispersion of the disk giants, $82 \pm 21 \mathrm{~km} \mathrm{~s}^{-1}$, is lower than that of the clump/RHB stars but higher than Lewis \& Freeman's (1989) predicted value of $\approx 72$ $\mathrm{km} \mathrm{s}^{-1}$ for the velocity dispersion of old disk stars at the mean distance of our disk sample; it is possible that the higher velocity dispersion indicates that there are some stars in the metal-rich end of the thick disk in our sample at low distances. Sharples et al. (1990) noted that the bright M giants toward BW had a lower velocity dispersion than the bulk of the sample, and attributed these stars to the foreground disk. They found $\sigma\left(v_{r}\right)=71_{-11}^{+20} \mathrm{~km}$

$\mathrm{s}^{-1}$ for the bright stars, consistent within the errors to our value for the disk subsample in this survey.

\subsubsection{Halo stars}

The remaining 15 stars in our sample $(5 \%)$ have $\mathrm{R}>10 \mathrm{kpc}$, and so appear to lie beyond the bulge. As mentioned earlier, we would not expect to see disk stars at such large distances because the line-of-sight is so far from the plane. These distant stars have a low mean abundance and a high velocity dispersion, suggesting that many of them belong to the Galactic halo. It is also possible that some are bulge stars with much higher reddening than average (and therefore whose distance is over-estimated), but this would require that some small patches in BW have $E_{V-I}$ which is up to $\sim 0.5 \mathrm{mag}$ higher than the average value.

\subsection{The Distance Distribution in Baade's window}


Table 1 lists the estimated distance for each star, and a classification as bulge clump/RHB (C), bulge giant branch (B), disk giant branch (D) or halo giant $(\mathrm{H})$. In the discussion which follows, the term 'bulge stars' refers to the combined $\mathrm{B}+\mathrm{C}$ sample which contains 268 stars, i.e. $83 \%$ of the stars with metallicity estimates. Table 4 summarizes the basic properties of the $\mathrm{C}, \mathrm{B}, \mathrm{D}$ and $\mathrm{H}$ subsamples.

Figure 8 shows the distribution of estimated distances for the entire sample of 322 stars (dashed line), as well as the distribution with with the clump/RHB stars excluded (solid line). The latter distribution is more representative of the distance distribution in the BW sample because of the systematic uncertainty in the distance estimates for clump/RHB stars. This $\mathrm{B}+\mathrm{D}+\mathrm{H}$ subsample has a mean distance of $6.6 \mathrm{kpc}$ and a median distance of $6.4 \mathrm{kpc}$. This is slightly lower than our adopted Galactic Center distance of $7.5 \mathrm{kpc}$ both because of the inclusion of foreground disk giants and because the magnitude cutoff of the proper motion sample means that we may not see the most distant of the metal-rich bulge stars.

We see a slight excess of disk giants in the $\mathrm{R}=2-3 \mathrm{kpc}$ bin in Figure 8 . A similar feature was noted by by P94 in their study of CMDs in BW (cf. Terndrup 1988), and attributed to spiral arm structure in the galactic disk.

As mentioned above, our distance estimates for the clump/RHB stars will be systematically low because these stars lie $\sim 0.5-0.7 \mathrm{mag}$ above the giant branch stars of similar color in the CMD (Terndrup 1988; P94; Ng et al. 1995). The mean distances for the $\mathrm{B}$ and $\mathrm{C}$ subsamples, 7.1 and $5.7 \mathrm{kpc}$, respectively, confirm this. The difference in distance modulus for the mean of two samples is $0.48 \mathrm{mag}$, consistent from the difference expected from the CMDs in the bulge. In Figure 8 we present an alternate distance distribution for our sample, which was constructed by arbitrarily increasing the distances of the $\mathrm{C}$ group by a factor of 1.25 to bring the mean distance equal to that of the B subsample. The resulting distribution is shown in Figure 9. The mean and median distances are, respectively, 7.6 kpc and $6.9 \mathrm{kpc}$.

Figure 10 shows the observed contribution of each subgroup to the BW sample as a function of $V$ magnitude. Foreground disk giants contribute roughly $50 \%$ of the sample at $V=15,20 \%$ at $V=16$ and are almost absent by $V=17$. Bulge clump stars occupy a narrow range between $V=16.5$ and 17.5 .

\section{The abundance distribution of $K$ giants in Baade's Window}




\subsection{The $[\mathrm{Fe} / \mathrm{H}]$ distribution}

Figure 11 shows a histogram of $[\mathrm{Fe} / \mathrm{H}]$ for the 268 bulge stars with abundance measurements (classes $\mathrm{B}$ and $\mathrm{C}$ above). The weighted mean abundance for this group is $\langle[\mathrm{Fe} / \mathrm{H}]\rangle=-0.11 \pm 0.04$, with a dispersion of $\sigma([\mathrm{Fe} / \mathrm{H}])=0.46 \pm 0.06$ dex; the median abundance is -0.03 dex. Over half the stars lie in the range $[\mathrm{Fe} / \mathrm{H}]=-0.4$ to +0.3 .

A potential problem with the derivation of abundances from our low-resolution spectra is that the FFBG formulation is only valid for $-0.8 \leq[\mathrm{Fe} / \mathrm{H}] \leq+0.5$. Above $[\mathrm{Fe} / \mathrm{H}]$ +0.5 the abundance estimates are extrapolations, while below $[\mathrm{Fe} / \mathrm{H}]-0.8$ the iron lines measured by the $\langle\mathrm{Fe}\rangle$ index become too weak to yield reliable results. About $18 \%$ of our sample have derived $[\mathrm{Fe} / \mathrm{H}]$ values which lie outside this range of validity, but estimates of the mean abundance in our sample are unlikely to be seriously affected by their inclusion.

The mean abundance for our sample of 268 bulge $\mathrm{K}$ giants is somewhat higher than the value of $\langle[\mathrm{Fe} / \mathrm{H}]\rangle=-0.25$ derived by MR94 from a recalibration of the 88 giants observed by $\mathrm{R} 88$. If we compare our $[\mathrm{Fe} / \mathrm{H}]$ with the recalibrated $[\mathrm{Fe} / \mathrm{H}]$ for the $\mathrm{R} 88$ stars in common with our sample using equation (6) of MR94, we find

$$
\left\langle[\mathrm{Fe} / \mathrm{H}]_{\mathrm{SRT}}-[\mathrm{Fe} / \mathrm{H}]_{\mathrm{R} 88^{\prime}}\right\rangle=0.14 \pm 0.23 \text { (s.d.), }
$$

where the recalibrated Rich (1988) scale as denoted R88'. Almost all of this difference is from stars with $[\mathrm{Fe} / \mathrm{H}]>+0.3$, for which in comparison to the echelle metallicities in BW (see above) our calibration is possibly too high. Taking stars with $[\mathrm{Fe} / \mathrm{H}] \leq+0.3$ in our solution only, we find

$$
\left\langle[\mathrm{Fe} / \mathrm{H}]_{\mathrm{SRT}}-[\mathrm{Fe} / \mathrm{H}]_{\mathrm{R} 88^{\prime}}\right\rangle=0.02 \pm 0.15 \text { (s.d.) }
$$

Thus although our measured values of $[\mathrm{Fe} / \mathrm{H}]$ agree well with those measured by MR94 for the few stars we have in common, it appears that our $[\mathrm{Fe} / \mathrm{H}]$ scale could be systematically higher by $\sim 0.15$ dex than the R88 scale as recalibrated by MR94, and that the difference is primarily because we may be overestimating the metallicities at the top end. This difference is about equal to that found by comparison to the results of high-resolution studies in BW (Sec. 2.4, above). However, we do not think it appropriate to recalibrate our $[\mathrm{Fe} / \mathrm{H}]$ measurements at this stage. The MR94 recalibration of the R88 scale is based on only 11 stars in common, and a larger sample of good-quality echelle spectra is clearly needed to set the low-resolution abundance measurements on a firm basis. For now, we simply accept that the zero point of our $[\mathrm{Fe} / \mathrm{H}]$ scale is uncertain by 0.10 to 0.15 dex, and bear this in mind in the discussion which follows. 


\subsection{Abundance measurements from $\mathrm{Mg}$ and $\mathrm{CN}$ indices}

As well as deriving $[\mathrm{Fe} / \mathrm{H}]$ from the $\langle\mathrm{Fe}\rangle$ index, we can use the $\mathrm{Mg}_{2}$ and $\mathrm{CN}$ indices as alternative abundance indicators. If the ratios $[\mathrm{Mg} / \mathrm{Fe}]$ and $[(\mathrm{C}+\mathrm{N}) / \mathrm{Fe}]$ have the same values in BW stars as in the Sun (as the FFBG relations assume), then we should derive the same mean abundance for $[\mathrm{Mg} / \mathrm{H}]$ and $[\mathrm{CN} / \mathrm{H}]$ as for $[\mathrm{Fe} / \mathrm{H}]$ in the sample as a whole. There are, however, reasons to expect non-solar abundance ratios in many of our BW giants. Old stellar populations in the Galaxy generally have $[\mathrm{Mg} / \mathrm{Fe}]>0$, at least for abundances well below solar (e.g., Lambert 1987; Wheeler et al. 1989). In BW, there is evidence from both low-resolution spectra (Paper I) and echelle studies (MR94) for an enhancement of [Mg/Fe] in bulge giants. The $\mathrm{Mg}$ and Fe line strengths in the integrated light of elliptical galaxies and spiral bulges also show evidence for a Mg overabundance (Worthey et al. 1992).

Once again, we use the relations tabulated by FFBG to convert our measured line-strength indices to abundances. By doing this, we are implicitly assuming that the BW giants have the same values of gravity as a function of temperature as the calibrating stars (mostly solar-neighborhood giants) in FFBG; using the G93 formulations, with different assumptions about the gravities of the BW giants, gives somewhat different results which we will discuss below (Sec. 4.5). We calculated abundances from the $\mathrm{Mg}_{2}$ and $\mathrm{CN}$ indices] for all stars where these indices were measurable, but chose to analyze only the set of 195 stars for which the errors in all three abundance measurements were less than 0.5 dex. Even for these stars, the errors in the individual abundance ratios $[\mathrm{Mg} / \mathrm{Fe}]$ and $[\mathrm{CN} / \mathrm{Fe}]$ are quite high (typically 0.4 to $0.5 \mathrm{dex}$ ), but the sample as a whole shows some interesting trends which are worth discussing. Table 5 summarizes the mean abundance ratios for several subgroups.

Figure 12 shows the FFBG calibration of abundance for $\mathrm{Mg}_{2}$ and $\mathrm{CN}$ as a function of $T_{\mathrm{e}}$ (compare to Figure 5). The $\mathrm{Mg}_{2}$ index is strongly temperature-sensitive, while the $\mathrm{CN}$ index is almost independent of temperature for $\mathrm{K}$ giants. It is clear from Figure 12 that the BW stars in our sample do not have the same abundances on the three scales: the mean $[\mathrm{Mg} / \mathrm{H}]$ is near solar, as was $[\mathrm{Fe} / \mathrm{H}]$, but the mean $[\mathrm{CN} / \mathrm{H}]$ is considerably lower, with $\langle[\mathrm{CN} / \mathrm{H}]\rangle \sim-0.3$.

\footnotetext{
${ }^{7}$ The abundances from the $\mathrm{Mg}_{2}$ and $\mathrm{CN}$ indices will be denoted $[\mathrm{Mg} / \mathrm{H}]$ and $[\mathrm{CN} / \mathrm{H}]$, and the relative abundances with respect to the metallicities from $\langle\mathrm{Fe}\rangle$ will be denoted $[\mathrm{Mg} / \mathrm{Fe}]$ and $[\mathrm{CN} / \mathrm{Fe}]$. The reader is reminded that we are not deriving abundance ratios in any true sense. We are comparing metallicity indicators from different features; these probably should have been called $\mathrm{M}(\langle\mathrm{Fe}\rangle), \mathrm{M}\left(\mathrm{Mg}_{2}\right)$, and $\mathrm{M}(\mathrm{CN})$. Our notation for the abundance ratio is a convenient shorthand: e.g., $[\mathrm{CN} / \mathrm{Fe}]=\mathrm{M}(\mathrm{CN})-\mathrm{M}(\langle\mathrm{Fe}\rangle)$.
} 


\section{3. $[\mathrm{Mg} / \mathrm{H}]$}

Figure 13 (lower) shows a histogram of $[\mathrm{Mg} / \mathrm{H}]$ for the 159 bulge stars in the $(\mathrm{B}+\mathrm{C})$ groups which have reasonably small errors from all three indices. The distribution is similar to that of $[\mathrm{Fe} / \mathrm{H}]$ in Figure 5. Both the $[\mathrm{Mg} / \mathrm{H}]$ and $[\mathrm{Fe} / \mathrm{H}]$ distributions have a dispersion of $0.50 \mathrm{dex}$. The mean value of $[\mathrm{Mg} / \mathrm{Fe}]$ for this group is $0.08 \pm 0.03 \mathrm{dex}$.

If the bulge sample is divided at $[\mathrm{Fe} / \mathrm{H}]=0.0$, the metal-poor stars have a higher mean value of $[\mathrm{Mg} / \mathrm{Fe}]$ than the metal-rich stars (see Table 5). Figure 14 (lower) shows the relation between $[\mathrm{Mg} / \mathrm{Fe}]$ and $[\mathrm{Fe} / \mathrm{H}]$ for individual bulge stars. Stars with $[\mathrm{Fe} / \mathrm{H}]>0$ show no strong Mg excess, and the behavior of the bulge stars as a whole appears similar to that of stars in the old disk and halo (e.g., Lambert 1987).

The Mg overabundances which we derive are modest, and lower than our estimate of $\sim+0.3$ dex in Paper I based on plots of line-strength indices and some simple models. They are also lower than the typical values measured by MR94. For the 5 stars in common (our measurement of $[\mathrm{Mg} / \mathrm{H}]$ for star $3-152$ is unreliable), we find $\langle[\mathrm{Mg} / \mathrm{Fe}]\rangle=+0.03 \pm 0.06$, significantly lower than the value of $+0.31 \pm 0.05$ measured by MR94. For 49 stars in the range $[\mathrm{Fe} / \mathrm{H}]-0.5$ to 0.0 , we find $\langle[\mathrm{Mg} / \mathrm{Fe}]\rangle=+0.11 \pm 0.06$, compared to typical values of +0.4 dex measured by $\mathrm{MR}$ for stars in the same $[\mathrm{Fe} / \mathrm{H}]$ range.

\section{4. $[\mathrm{CN} / \mathrm{H}]$}

The behavior of $[\mathrm{CN} / \mathrm{H}]$ shows several puzzling features. At first sight, $\mathrm{CN}$ is potentially the most robust of the line-strength indices we have measured, since (Figure 12, top panel), it is almost independent of temperature for $\mathrm{K}$ giants and thus is unaffected by small errors in the assumed temperature or reddening. Detailed examination of the CN strengths in field and cluster $\mathrm{K}$ giants, however, shows that $\mathrm{CN}$ is affected by other factors, most likely core/surface mixing (G93 and references therein).

The distribution of $[\mathrm{CN} / \mathrm{H}]$ in Figure 13 is narrower than that for $[\mathrm{Mg} / \mathrm{H}]$ and $[\mathrm{Fe} / \mathrm{H}]$ (see Figure 2), and peaks at a significantly lower abundance. This is surprising, since mixing would be expected to increase the observed abundance of CNO elements and differences in the amount of mixing along the giant branch might be expected to broaden the observed $[\mathrm{CN} / \mathrm{H}]$ distribution so that it was wider than that of $[\mathrm{Fe} / \mathrm{H}]$.

For the metal-poor and metal-rich subgroups of our bulge stars, the average $[\mathrm{CN} / \mathrm{Fe}]$ values are very different. The metal-poor stars (Table 5) have a mean $[\mathrm{CN} / \mathrm{Fe}]$ ratio close 
to solar, while the metal-rich stars have $\langle[\mathrm{CN} / \mathrm{Fe}]\rangle=-0.47 \pm 0.04$. These mean values are plotted with the individual determinations of $[\mathrm{CN} / \mathrm{Fe}]$ on the top panel of Figure 14.

R88 also noted that CN was weak relative to Fe in many of the BW stars in his sample (see also Janes 1975). He thought that surface gravity effects were unlikely to be the cause, since the $\mathrm{Mg}_{2}$ index (which, like CN, is gravity-sensitive) showed no tendency for CN-weak stars to have the higher gravities which would be needed to depress the CN band.

We considered the possibility that $[\mathrm{CN} / \mathrm{H}]$ reflects the 'true' abundance distribution of BW giants (i.e., a narrow range around a mean abundance of -0.3 dex, or 0.6 times solar), and that the derived $[\mathrm{Mg} / \mathrm{H}]$ and $[\mathrm{Fe} / \mathrm{H}]$ values are distorted by both patchy reddening in BW (which would broaden the distribution) and an assumed mean reddening which is too high. This appears unlikely. Comparing the observed $V-I$ color and $\mathrm{H} \beta$ line index (as described in Paper I) for stars with low and high [CN/Fe] shows no evidence for a difference in reddening between the two groups.

\subsection{Exploring the effects of gravity}

As mentioned earlier, a fixed relation between temperature and surface gravity is implicit in the FFBG calibration which we use to convert our line-strength measurements to abundances. Since bulge $\mathrm{K}$ giants may not necessarily follow the same $\log g-T_{\mathrm{e}}$ relation as those in the solar neighborhood, we explored the effects of this assumption by recomputing $[\mathrm{Fe} / \mathrm{H}],[\mathrm{Mg} / \mathrm{Fe}]$, and $[\mathrm{CN} / \mathrm{Fe}]$ from the G93 formalism, which explicitly includes both metallicity and gravity in its "fitting functions" for each line-strength index.

We first estimated $\log g$ and $V-K$ for each star (the latter is the G93 temperature indicator). For gravity, we made an initial estimate by assuming that each star was at a distance of $R=7.5 \mathrm{kpc}$, and derived $\log g$ from

$$
\log \left(g / g_{\odot}\right)=\log \left(M / M_{\odot}\right)-\log \left(L / L_{\odot}\right)+4 \log \left(T / T_{\odot}\right)
$$

where $M, L$, and $T$ are, respectively, the stellar mass, luminosity and temperature. We assumed $M=1.1 M_{\odot}$, a value appropriate for moderately old stars (this will be lower than the values implicit in the FFBG approach), and derived $T$ from the $V-I$ color as above. The luminosity was derived from the dereddened $I$ magnitude with bolometric corrections appropriate for bulge stars (Tiede et al. 1995), and the $V-K$ color was derived from the dereddened $V-I$ using a polynomial relation derived from a small set of stars in BW which 
have both colors (Tiede et al. 1995; Paper I). We then estimated [Fe/H] for each star using the mean of the values from the G93 fitting functions for the Fe 5270 and Fe 5335 indices.

The $[\mathrm{Fe} / \mathrm{H}]$ estimates derived in this way agree well with our earlier determinations using the FFBG relations. The G93 values are tightly correlated with the FFBG values (as expected, since they are derived from the same indices), but $\approx 0.05$ dex higher. With these new $[\mathrm{Fe} / \mathrm{H}]$ values we computed the distance to each star as described earlier, which led to a new estimate of $L$ and hence $\log g$. These were then inserted into the fitting functions for the $\mathrm{Mg}_{2}$ and $\mathrm{CN}$ indices to derive estimates of $[\mathrm{Mg} / \mathrm{H}]$ and $[\mathrm{CN} / \mathrm{H}]$. These were differenced with $[\mathrm{Fe} / \mathrm{H}]$ to provide $[\mathrm{Mg} / \mathrm{Fe}]$ and $[\mathrm{CN} / \mathrm{Fe}]$. The earlier remarks on the large errors in these quantities apply to this computation as well.

Finally, we divided the sample into disk, bulge, and halo groups as described earlier in Sec. 3 and computed mean values of $[\mathrm{Mg} / \mathrm{Fe}]$ and $[\mathrm{CN} / \mathrm{Fe}]$ for each group. These are listed in Table 5 together with the earlier values from the FFBG approach.

In Figure 15 we display the derived mean values of (lower panel) $\langle[\mathrm{Mg} / \mathrm{Fe}]\rangle$ and (upper panel) $\langle[\mathrm{CN} / \mathrm{Fe}]\rangle$ as a function of $[\mathrm{Fe} / \mathrm{H}]$ for the stars in the bulge $(\mathrm{B}+\mathrm{C})$ sample. These were computed by sorting the stars by $[\mathrm{Fe} / \mathrm{H}]$, and dividing the stars into four bins of equal numbers. In both panels of Figure 15, the mean abundance ratios as computed in the FFBG approach are shown as open points with error bars (these are errors in the mean). The filled points connected with the dashed line are the mean abundance ratios from the G93 formalism. In the lower panel, we also show (solid line) the trend in the relative abundances of the alpha-capture elements in the local disk and halo (e.g., Wheeler et al. [1989). The plusses show the derived $[\mathrm{Mg} / \mathrm{Fe}]$ ratios for individual K giants from MR94.

For $\mathrm{CN}$, the two approaches give similar results. In both cases, we find $\langle[\mathrm{CN} / \mathrm{Fe}]\rangle \approx 0$ for the most metal-poor stars in our sample, and $\langle[\mathrm{CN} / \mathrm{Fe}]\rangle \approx-0.4$ above $[\mathrm{Fe} / \mathrm{H}]=0.0$. Thus the low abundances derived from the CN index for metal-rich stars in our sample do not appear to be related to surface-gravity effects.

For $\mathrm{Mg}$, the G93 formalism gives $[\mathrm{Mg} / \mathrm{Fe}]$ values up to 0.3 dex higher than the FFBG approach. As G93 showed, the $\mathrm{Mg}_{2}$ index is strongly affected by surface gravity while the effect on the strengths of the Fe lines is negligible. In the FFBG approach, the metallicity calibration of the $\mathrm{Mg}_{2}$ index was made under assumption that $[\mathrm{Fe} / \mathrm{H}] \equiv[\mathrm{Mg} / \mathrm{H}]$, using adopted metallicities from $\mathrm{K}$ giants in the solar neighborhood. If these stars are significantly younger than $\mathrm{K}$ giants in the bulge, they would have higher surface gravities (and therefore stronger $\mathrm{Mg}$ absorption lines) at a given luminosity. For the G93 calculation of [Mg/Fe], we assumed a lower gravity (appropriate for relatively old stars in the bulge), which means that we would derive higher $[\mathrm{Mg} / \mathrm{Fe}]$ to match the observed $\mathrm{Mg}_{2}$ values in our sample (the 
$\mathrm{Mg}_{2}$ index increases with metallicity and with gravity).

That the $\mathrm{K}$ giants in the solar neighborhood probably have higher gravities than bulge stars may be seen directly in Figure 3 of G93, which plots $\log g$ versus temperature for their calibration stars. At colors typical of our sample, the gravities of stars in old globular clusters like M 3 and M92 are $~ 1.5$ dex lower than those for stars in metal-rich disk clusters like M 67 and M 71. We derive higher values of $[\mathrm{Mg} / \mathrm{Fe}]$ (and better agreement with the results of MR94) if we use the G93 fitting functions and assume that the BW giants are substantially older than solar neighborhood K giants, i.e., their gravities are lower than for the $\mathrm{K}$ giants in the FFBG formulation. It is clear that the values of $[\mathrm{Mg} / \mathrm{Fe}]$ derived from low-resolution spectra are strongly dependent on assumptions about the surface gravities of bulge $\mathrm{K}$ giants, which cannot be measured directly from the low-resolution data (see also Figure 11 of Paper I).

\section{Summary and Discussion}

In this paper, the second in our series on the kinematics and abundances of $\mathrm{K}$ giants in the Baade's Window field of the Galactic bulge, we have used Fe line strengths to measure $[\mathrm{Fe} / \mathrm{H}]$ for 322 stars. The derived abundance scale is close to that from two recent studies of bulge stars at high resolution. In preparation for our upcoming analysis of the proper motions and radial velocities of our sample, we derived photometric parallaxes and showed that the line-of-sight towards BW contains subgroups which we can identify with the foreground disk, clump and red giant stars in the bulge, and distant halo stars. About $80 \%$ of our sample can be classified as bulge giant-branch or clump/RHB stars.

There has been considerable discussion in the literature about the mean metallicity in the bulge. Several earlier studies, based on line strengths in K giants (Whitford \& Rich 1983; Rich 1988), Washington photometry (Geisler \& Friel 1992) and the photometric and spectroscopic properties of M giants (Frogel \& Whitford 1987; Frogel et al. 1990; Terndrup et al. 1990; Sharples et al. 1990; Terndrup et al. 1991) concluded that the mean abundance of the stars in BW was $\sim+0.2$. This was revised sharply downward to $\langle[\mathrm{Fe} / \mathrm{H}]\rangle \approx-0.25$ by MR94 based on their high-resolution studies of $11 \mathrm{~K}$ giants in BW, and a recent study of the infrared color-magnitude diagram in BW (Tiede et al. 1995) yields much the same result. MR94 give a lengthy discussion of why their estimated metallicities are lower, concluding that the R88 scale measured $[(\mathrm{Mg}+\mathrm{Fe}) / \mathrm{H}]$, with $[\mathrm{Mg} / \mathrm{Fe}]>0$ in the bulge; they also found elevated relative abundances of $\mathrm{Ti}$ in their sample, and speculated that if 
this were the case for the bulge $\mathrm{M}$ giants, the average abundances found for these stars would be higher than those from Fe.

In this paper, we find $\langle[\mathrm{Fe} / \mathrm{H}]\rangle=-0.11 \pm 0.03$ for our combined sample of bulge giants and clump/RHB stars. Our $[\mathrm{Fe} / \mathrm{H}]$ measurements agree well with those measured at high resolution for the few stars in common; but appear to be $\sim 0.15$ dex higher than the $\mathrm{R} 88$ scale as recalibrated by MR94, though much closer for stars with $[\mathrm{Fe} / \mathrm{H}] \leq+0.3$. Because the recalibration is based on a small number of stars we have chosen not to apply any correction to our measured $[\mathrm{Fe} / \mathrm{H}]$ values at this stage.

We then explored whether the metallicity scales derived from the $\mathrm{CN}$ and $\mathrm{Mg}_{2}$ indices are the same as those from the Fe features. There is evidence that the metal-poor half of our sample has $[\mathrm{Mg} / \mathrm{Fe}]>0$, but in general the Mg excess is less than that found by MR94. The FFBG formalism which we used to derive $[\mathrm{Mg} / \mathrm{Fe}]$ from our low-resolution spectra assumes that bulge $\mathrm{K}$ giants have the same surface gravity as giants of similar abundance in the local disk. This is unlikely to be the case if most bulge giants are relatively old (see also Paper I), so it is possible that we have underestimated $[\mathrm{Mg} / \mathrm{Fe}]$ for many of our stars.

The behavior of CN is puzzling - many of our stars, particularly the the most metal-rich ones, appear to be $\mathrm{CN}$-weak compared to local giants. G93 discussed CN extensively, and argued that the primary controller for the $\mathrm{CN}$ index was mixing at the base of the giant branch. They found a strong rise in CN line strength beginning at about $V-K$ $=2.1$, and essentially complete by $V-K=2.2$. These colors correspond to the bluest stars in our sample, so that if the bulge stars have the same combination of metallicities and/or mixing as the $\mathrm{K}$ giants in the Lick program, we would expect to see similarly strong $\mathrm{CN}$ features. We find, however, near solar $[\mathrm{CN} / \mathrm{Fe}]$ for the metal-poor half of the bulge sample but weak $\mathrm{CN}$ for stars with $[\mathrm{Fe} / \mathrm{H}]>0$.

The weak CN lines suggest that bulge stars may differ in some respects from the stars which make up ellipticals and the bulges of other spirals. For Mg, Worthey et al. (1992) have shown that the nuclear spectra of most ellipticals have higher $\mathrm{Mg}_{2}$ indices compared to iron, which cannot be explained with models of the integrated light which have $[\mathrm{Mg} / \mathrm{Fe}]$ $=0$. They suggest that $\mathrm{Mg}$ is enhanced in many external systems, and that the $\mathrm{Mg}$ overabundance increases with galaxy luminosity. In Galactic bulge giants, CN appears to be much weaker than in the bulge of M31, which has a high CN index (Worthey 1994). The behavior of CN in external galaxies is not well understood. For example the Worthey models at the highest metallicity $([\mathrm{Fe} / \mathrm{H}]=+0.3)$ always produce much stronger indices than observed in galaxies, suggesting that the mean abundance is less than in the models. The exception to this is $\mathrm{CN}$, where the observed line strength can be higher than in any model, suggesting that it is not just the overall metallicity which varies. 
We wish to thank Gary DaCosta, Ken Freeman, Jeremy Mould, John Norris and Ruth Peterson for advice and helpful discussions. RMR received support from NASA Grant NAGW 2479. DT received support from the National Science Foundation through grants AST-9157038 and INT-9215844. We are grateful to an anonymous referee for several valuable comments on the original version of this paper. 


\section{REFERENCES}

Armandroff, T. E., \& DaCosta, G. S. 1986, AJ, 92, 777

Barbuy, B., Castro, S., Ortolani, S., \& Bica, E. 1992, A\&A, 259, 607

Bell, R. A., \& Gustafsson, B. 1989, MNRAS, 236, 653

Bessell, M. S. 1979, PASP, 91, 589

Bessell, M. S., Brett, J. M., Sholz, M., \& Wood, P. R. 1989, A\&AS, 77, 1

Bessell, M. S., Pfitzner, D., \& Sadler, E. M. 1995, in preparation

Bica, E., Barbuy, B., \& Ortolani, S. 1991, ApJ, 382, L15

Blanco, B. M. 1984, AJ, 89, 1836

Blanco, V. M., McCarthy, M. F., \& Blanco, B. M. 1984, AJ, 89, 636

Blanco, V. M., \& Terndrup, D. M. 1989, AJ, 98, 843

Castro, S., Barbuy, B., Bica, E., Ortolani, S., \& Renzini, A. 1995, A\&AS, 111, 17

Da Costa, G. S., \& Armandroff, T. E. 1990, AJ, 100, 162

Faber, S. M., Friel, E. D., Burstein, D., \& Gaskell, C. M. 1985, ApJS, 57, 711 (FFBG)

Frogel, J. A., Terndrup, D. M., Blanco, V. M., \& Whitford, A. E. 1990, ApJ, 357, 453

Frogel, J. A., \& Whitford, A. E. 1987, ApJ, 320, 199

Geisler, D., \& Friel, E. D. 1992, AJ, 104, 128 (GF92)

Gorgas, J., Faber, S. M., Burstein, D., González, J., Courteau, S., \& Prosser, C. 1993, ApJS, 86, 153 (G93)

Green, E. M., Demarque, P., \& King, C. R. 1987, The Revised Yale Isochrones and Luminosity Functions, Yale University Observatory

Habing, H. J., Onlon, F., Chester, T., Gillett, F., Rowan-Robinson, M., \& Neugebauer, G. 1985, A\&A, 152, L1

Harmon, R., \& Gilmore, G. 1988, MNRAS, 235, 1025 
Izumiura, H., Deguchi, S., Hashimoto, O., Nakada, Y., Onaka, T., Ono, T., Ukita, N., \& Yamamura, I. 1995, ApJS, 98, 271

Janes, K. A. 1977, ApJ, 212, L59

Jones, J. B., Wyse, R. F. G., \& Gilmore, G. 1995, PASP, 107, 632

Kaluzny, J., \& Rucinski, S. M. 1995, A\&AS, 114, 1

Kent, S. M. 1992, ApJ, 387, 181

Lambert, D. L. 1987, JA\&A, 8, 103

Lewis, J. R., \& Freeman, K. C. 1989, AJ, 97, 139

McWilliam, A., \& Rich, R. M. 1994, ApJS, 91, 749 (MR94)

Mihalas, D., \& Binney, J. 1981, Galactic Astronomy (Freeman, San Francisco)

Montgomery, K. A., Janes, K. A., \& Phelps, R. L. 1995, AJ, 108, 585

Ng, Y.-K., Bertelli, G., Chiosi, C., \& Bressan, A. 1995, ApJ, 453, 837

Ortolani, S., Barbuy, B., \& Bica, E., 1990, A\&A, 236, 362

Paczynski, B., Stanek, K. Z., Udalski, A., Szmanski, M., Kaluzny, J., Kubiak, M., \& Mateo, M. 1994, AJ, 107, 2060

Rich, R. M. 1988, AJ, 95, 828 (R88)

Rich, R. M. 1990, ApJ, 362, 604

Ridgway, S. T., Joyce, R. R., White, N. M., \& Wing, R. F. 1980, ApJ, 235, 126

Sharples, R., Walker, A., \& Cropper, M. 1990, MNRAS, 246, 54

Spaenhauer, A., Jones, B.F., \& Whitford, A. E. 1992, AJ, 103, 297

Terndrup, D. M. 1988, AJ, 96, 884

Terndrup, D. M., Frogel, J. A., \& Whitford, A. E. 1990, ApJ, 357, 453

Terndrup, D. M., Frogel, J. A., \& Whitford, A. E., 1991, ApJ, 378, 742

Terndrup, D.M., Sadler, E.M., \& Rich, R.M. 1995 AJ, 110, 1774 (Paper I)

Tiede, G. P., Frogel, J. A., \& Terndrup, D. M. 1995, AJ, 110, 2788 
Trippico, M. J., Bell, R. A., Dorman, B., \& Hufnagel, B. 1995, AJ, 109, 1697

Wheeler, J.C., Sneden, C., \& Truran, J.W. 1989 ARA\&A, 27, 279

Weiland, J. L., et al. ApJ, 425, L81

Whitford, A. E., \& Rich, R. M. 1983, ApJ, 274, 723

Worthey, G., Faber, S. M., \& González, J. J., 1992, ApJ, 398, 69

Worthey, G. 1994, ApJS, 95, 107

Worthey, G., Faber, S. M., González, J. J., \& Burstein, D. 1994, ApJS, 94, 687 
Fig. 1.- Relation between the reddening-corrected color $(V-I)_{0}$ and effective temperature $T_{\mathrm{e}}$, from Bessell (1979).

Fig. 2.- Conversion between $T_{\mathrm{e}},\langle\mathrm{Fe}\rangle$ and $[\mathrm{Fe} / \mathrm{H}]$, adapted from Faber et al. (1985). The 322 individual data points with $T_{\mathrm{e}}$ in the range $3900-5160 \mathrm{~K}$ are also shown.

Fig. 3.- Comparison between $[\mathrm{Fe} / \mathrm{H}]$ measurements from this study and those measured by Rich (1988; lower panel) and Geisler \& Friel (1992; upper panel) for stars in common. The dotted lines show constant offsets of +0.2 and 0.15 dex respectively.

Fig. 4.- Comparison between $[\mathrm{Fe} / \mathrm{H}]$ measurements from this study, and measurements from high-resolution spectra studies by (filled circles) McWilliam \& Rich (1994) and (open circles) Bessell et al. (1995). The dashed line denotes identity.

Fig. 5.- Effect of changes in $\mathrm{E}_{V-I}$ on the measured $[\mathrm{Fe} / \mathrm{H}]$ value for stars of different temperatures.

Fig. 6.- Predicted observed $V$ magnitude for K0 and K5 giants (solid lines) and a K0 dwarf (dashed line) at various distances along the line-of-sight in BW.

Fig. 7.- Adopted absolute magnitude sequences. Shown in the left panel are isochrones for the four globular clusters described in the text (solid lines). The two dotted lines show the region in which we have assumed that the bulge clump stars lie. Open circles represent stars in our sample, assuming that all the stars are distance of $7.5 \mathrm{kpc}$. The right panel repeats the globular cluster sequences (thin solid lines) and the clump region (horizontal dotted lines). The thick solid line shows the giant branch for $[\mathrm{Fe} / \mathrm{H}]=+0.3$ as extrapolated from the other giant branches as described in the next. The adjacent dotted giant branch is for the Revised Yale Isochrone (Green et al. 1987) at $[\mathrm{Fe} / \mathrm{H}]=+0.3$ and an age of 10 Gyr. The points $(\times)$ represent the observed giant branch for the old metal-rich open cluster NGC 6791 (Kaluzny \& Rucinski 1995; Montgomery et al. 1995) shifted to the reddening and distance modulus advocated by Trippico et al. (1995), who derive $[\mathrm{Fe} / \mathrm{H}]=+0.36 \pm 0.12$ for NGC 6791 .

Fig. 8.- Distribution of estimated distances for the entire sample (dashed line), and for stars on the giant branch only, with clump stars excluded (solid line). The bar shows the typical error in an individual distance measurement. 
Fig. 9.- Alternative distance distribution for all stars in the survey, constructed by increasing the distances of the clump/RHB stars in Figure 7 by a factor of 1.25.

Fig. 10.- Relative contribution of bulge, disk and halo stars to the stellar population in BW, as a function of observed apparent $V$ magnitude.

Fig. 11. - Histogram of $[\mathrm{Fe} / \mathrm{H}]$ for the 268 stars which are members of the bulge (i.e. bulge giant-branch and clump stars).

Fig. 12.- Conversion between $T_{\mathrm{e}}$, line strength and abundance for the $\mathrm{Mg}_{2}$ and $\mathrm{CN}$ indices, adapted from Faber et al. (1985). As in Figure 2, the 322 individual data points with $T_{\mathrm{e}}$ in the range $3900-5160 \mathrm{~K}$ are also shown.

Fig. 13.- Histograms of $[\mathrm{Mg} / \mathrm{H}]$ (lower) and $[\mathrm{CN} / \mathrm{H}]$ (upper) for bulge stars in our sample.

Fig. 14. - Plots of the abundance ratios $[\mathrm{Mg} / \mathrm{Fe}]$ (lower) and $[\mathrm{CN} / \mathrm{Fe}]$ (upper) from the FFBG calibaration as a function of $[\mathrm{Fe} / \mathrm{H}]$ for individual stars (shown as crosses). The large open squares show the mean values for stars with $[\mathrm{Fe} / \mathrm{H}]<0$ and $\geq 0$, while the solid line shows the relation between the $\mathrm{Mg}$ and Fe abundances for local stars in the disk and halo (Lambert 1987).

Fig. 15. - Basic trends in abundances from the $\mathrm{Mg}_{2}$ index (lower panel) and $\mathrm{CN}$ index (upper panel). The bulge $(\mathrm{B}+\mathrm{C})$ stars have been split into four equal bins in $[\mathrm{Fe} / \mathrm{H}]$. The mean values from the simpler FFBG calibration (Faber et al. 1985) calibration are shown by open circles, while the filled circles show the mean $[\mathrm{CN} / \mathrm{Fe}]$ and $[\mathrm{Mg} / \mathrm{Fe}]$ abundances derived from the G93 (Gorgas et al. 1993) formalism. In the lower panel, plus signs denote individual $[\mathrm{Mg} / \mathrm{Fe}]$ values from the study by McWilliam \& Rich (1994) and (as in Figure 14) the solid line shows the $[\mathrm{Mg} / \mathrm{Fe}]$ trends in the local disk and halo (Lambert 1987). 
TABLE 1. Measurements of $[\mathrm{Fe} / \mathrm{H}]$ for Baade's Window K-giants.

TABLE 2. Comparison of $[\mathrm{Fe} / \mathrm{H}]$ measurements for stars in common with other studies.

TABLE 3. Cross-identification with stars observed by Geisler \& Friel (1992). Asterisks mark the three stars with highly discrepant $[\mathrm{Fe} / \mathrm{H}]$ meaurements.

TABLE 4. Mean abundance, and radial velocity dispersion, for subsamples of the BW population.

TABLE 5. Mean abundance ratios for BW subsamples. 